\title{
Éditorial
}

\section{La richesse des régions ${ }^{1}$ \\ Marc-Urbain Proulx ${ }^{2}$, CRDT, UQAC}

Au cours des deux dernières décennies, la vaste périphérie de la métropole Montréal et de la capitale Québec a contribué à près de $60 \%$ du PIB québécois. Historiquement beaucoup plus élevé, ce ratio régional a fortement diminué au fil de l'industrialisation du Québec dont les activités furent largement polarisées par Montréal. La part régionale du PIB redevient actuellement en croissance légère à la faveur de ladite déconcentration, notamment dans la frange de Montréal et Québec. Signalons qu'à tous les ans autour de $60 \%$ à $70 \%$ de l'ensemble des investissements industriels du Québec s'effectue en périphérie.

De fait, certaines zones de la périphérie immédiate s'inscrivent comme des systèmes de production particulièrement fertiles, en particulier les corridors de la Beauce et des Laurentides ainsi que les milieux de Gatineau, Drummond, Trois-Rivières, Grandby, Sherbrooke, Bécancour, Victoriaville, entre lesquels existent de très nombreux petits pôles d'activités économiques. Sises dans le sud-est du Québec, ces zones et pôles illustrent ensemble une forme territoriale globale tout à fait particulière. S'agit-il d'un $\operatorname{arc}^{3}$, un archipel $^{4}$, une métapole ${ }^{5}$, un croissant manufacturier ${ }^{6}$ ? Peu importe le libellé, l'important étant que les spécialistes reconnaissent que le nouveau jeu des forces centripètes et centrifuges compose actuellement un nouveau territoire de cohérence dans la vallée du SaintLaurent ${ }^{7}$. D'autres milieux davantage périphériques comme Rivière-du-Loup, Baie-Comeau, Saguenay, Sept-Îles et autres Val d'Or, se positionnent aussi fort bien au Québec en matière de production.

Il apparaît évident à notre observation que les régions contribuent substantiellement à l'économie du Québec. $\mathrm{Si}$ la production et l'investissement régionaux sont au rendez-vous, la périphérie québécoise s'avère néanmoins confrontée, selon notre lecture, à un triple problème.

\section{Limiter l’érosion}

Bref, les régions du Québec produisent de plus en plus de richesse, mais bénéficient de moins en moins des conséquentes retombées financières dont les fuites s'accélèrent. Ainsi nous devons poser sérieusement la question que voici : à l'ère de ladite mondialisation, quels moyens réels disposent les régions et le Québec
Sous l'angle de l'emploi d'abord, la technologie devenue essentielle à la productivité remplace de plus en plus les travailleurs dans les activités de production en étant rapidement adoptée dans les activités périphériques, notamment l'agriculture, la forêt, les mines. Ce qui limite en conséquence le nombre de ménages pouvant tirer un revenu dans les lieux périphériques. S'expliquent ainsi largement le taux de chômage élevé et l'exode des jeunes de plusieurs régions.

Le $2^{\text {ème }}$ problème régional réside dans la tendance lourde à la désappropriation des activités économiques. Traditionnellement limitée à la grande industrie dans les pâtes et papier, les mines et l'aluminium, la faible propriété locale des entreprises affecte progressivement la petite industrie, les commerces, les services. L'effritement de la propriété locale s'est en réalité tout à fait généralisé au cours des dernières décennies. Il s'inscrit en particulier par l'envahissement des Géants à succursales multiples dans les secteurs du bois d'œuvre, de la restauration, de la quincaillerie, de la comptabilité, de la mercerie, dont la propriété des entreprises échappe de plus en plus aux collectivités territoriales. Cette désappropriation croissante affaiblit l'entrepreneurship local et limite ainsi considérablement la capacité de croissance endogène des collectivités.

D'autant plus que ce phénomène de désappropriation en périphérie accentue aussi l'érosion des profits vers les sièges sociaux qui sont localisés certes à Montréal, mais à l'évidence de plus en plus à Toronto, New-York, Londres et bientôt Shanghai. Mondialisation oblige, Montréal doit se contenter désormais d'obtenir des sièges sociaux secondaires dans plusieurs secteurs pourtant importants pour l'économie du Québec. Le déclin de la fonction financière de cette métropole s'avère ainsi accentué. Cet affaiblissement du cumul de capitaux à Montréal affecte négativement son rôle de moteur économique joué pour tout le Québec. Ce qui représente de ce fait, le $3^{\text {ème }}$ problème régional majeur.

pour bénéficier davantage de la croissante richesse créée dans la vaste périphérie?

En guise de réponse, une politique publique vigoureuse s'avère souhaitée. Certains avancent l'option concernée par l'octroi conditionnel (entretien routier, 
aménagement forestier, etc) à des instances territoriales, des redevances versées pour compenser l'exploitation des ressources naturelles. D'autres analystes proposent la mise en œuvre d'un instrument décentralisé pour favoriser et supporter l'acquisition de propriétés (énergie renouvelable, bois d'œuvre, transport, etc.) par les autorités territoriales. D'autres observateurs encore considèrent le nécessaire renouvellement de la planification territoriale davantage instrumentée, notamment en matière de montage de la faisabilité multicritère de projets innovateurs.

Cette question des moyens pour mieux maîtriser la richesse créée en régions devient d'autant plus pertinente puisque la périphérie du Québec maintiendra sûrement sa contribution substantielle au PIB dans le futur. D'abord, parce que les industries libérées par le transport routier préfèrent désormais se localiser hors des grands centres urbains comme Québec et Montréal.

\section{Pour un nouveau modèle}

L'importante contribution de la périphérie à la création de richesse au Québec plaide pour une politique publique qui puisse intervenir, de manière stratégique, au-delà du saupoudrage de nombreuses petites mesures clientélistes. L'appropriation territoriale de leviers économiques doit en réalité devenir prioritaire.

À cet effet, Québec pourrait à notre avis se doter d'une vision globale de l'imbrication géo-économique de ses divers territoires urbains, régionaux, métropolitains, ruraux, nordiques, etc. Il est devenu très clair que les modèles du passé ne conviennent plus pour saisir les interdépendances, les complémentarités et la concurrence entre les multiples territoires dans le contexte dynamique contemporain. D'une telle vision globale, les formes territoriales exprimées par les activités économiques illustreraient alors leur pertinence, notamment les agglomérations urbaines, les territoires de solidarité communautaire, les zones à usage ciblé, les corridors de développement. Sur ces territoires pertinents par leur intérêt collectif, leur
Ensuite, parce que les efforts pour ajouter de la valeur aux ressources naturelles portent fruits en périphérie dans les niches du bois, de l'aluminium, des produits de la mer, de l'eau, de l'agroalimentaire. Finalement, parce que les grands chantiers tels que ceux de Péribonka IV, Eastman, Baie-Comeau, La Romaine, Jonquière vont se multiplier encore à Caniapiscau, à Bécancour, à Alma, aux Monts-Ostih, à Cacouna, en Ungava. Car des bassins miniers, des gisements éoliens et des réserves hydrographiques offriront leur richesse à exploiter, en respect de l'environnement et des droits autochtones. Et que dire de la forêt boréale en constante régénération salutaire pour livrer de la fibre bien sûr, mais aussi du précieux carbone et de la biomasse à valoriser ? Cette forêt offre des gains potentiels considérables de productivité, notamment dans ses landes improductives à jardiner. cohérence, leur dynamisme, une procédure de planification innovatrice pourrait lancer un processus de mobilisation vers l'élaboration de projets territoriaux pouvant conduire à l'appropriation collective de nouveaux instruments pour lutter contre l'érosion de la richesse.

Sous l'angle de ces nouveaux instruments, le Québec ne part pas de zéro. Certaines expériences peuvent inspirer les territoires, notamment les actifs actuels des municipalités en matière hydroélectrique, commerciale, foncière, récréotouristique. Aussi, la formule coopérative illustre des succès territoriaux fort intéressants dans les domaines de la forêt, de la pêche, de l'éolien, de l'agriculture, de l'habitat. La multiplication récente des partenariats public - privé offre aussi un riche champ d'expérimentation. En réalité dans cette voie incontournable de l'appropriation territoriale en périphérie, le Québec est à l'heure de l'innovation institutionnelle territoriale dans un esprit de responsabilisation collective.

\section{Notes et références}

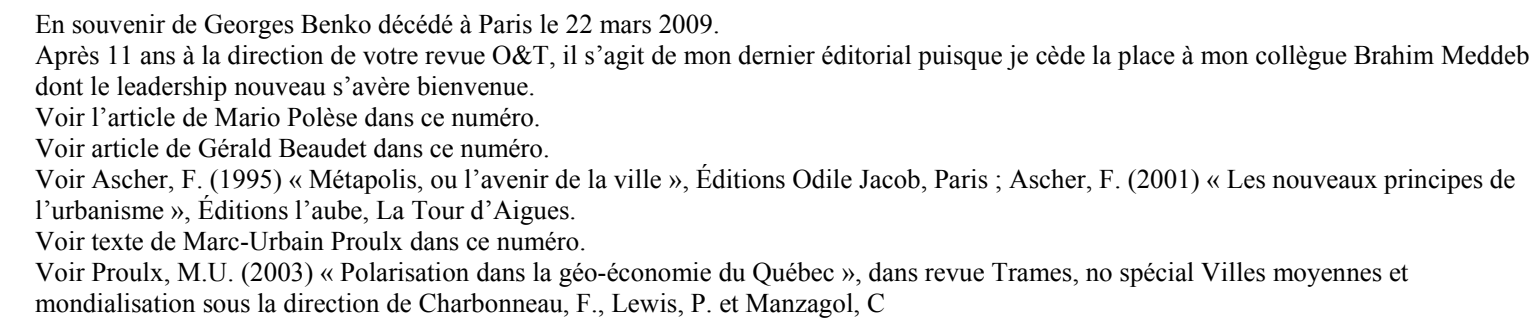

